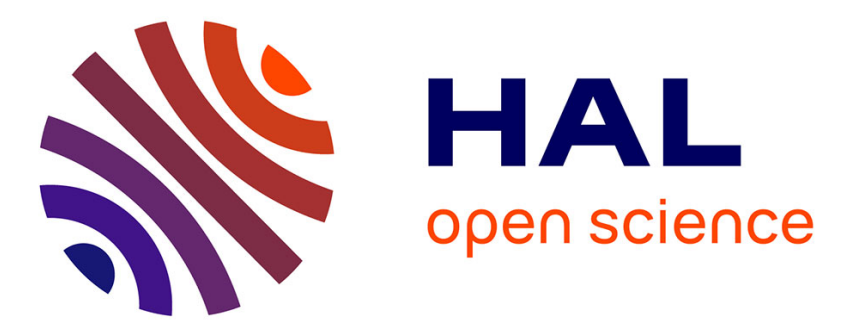

\title{
Arterial blood pressure analysis based on scattering transform II
}

Taous-Meriem Laleg, Claire Médigue, François Cottin, Michel Sorine

\section{To cite this version:}

Taous-Meriem Laleg, Claire Médigue, François Cottin, Michel Sorine. Arterial blood pressure analysis based on scattering transform II. 29th Annual International Conference Sciences and Technologies for Health - EMBC 2007, IEEE Engineering in Medicine and Biology Society, Aug 2007, Lyon / France. inria-00139529v2

\section{HAL Id: inria-00139529 \\ https://inria.hal.science/inria-00139529v2}

Submitted on 20 Jun 2007

HAL is a multi-disciplinary open access archive for the deposit and dissemination of scientific research documents, whether they are published or not. The documents may come from teaching and research institutions in France or abroad, or from public or private research centers.
L'archive ouverte pluridisciplinaire HAL, est destinée au dépôt et à la diffusion de documents scientifiques de niveau recherche, publiés ou non, émanant des établissements d'enseignement et de recherche français ou étrangers, des laboratoires publics ou privés. 


\title{
Arterial blood pressure analysis based on scattering transform II
}

\author{
Taous-Meriem Laleg, Claire Médigue, François Cottin and Michel Sorine
}

\begin{abstract}
Arterial blood pressure analysis based on scattering transform I" introduces a new method based on the scattering transform for a one dimensional Schrödinger equation to reconstruct the arterial blood pressure waves and separate its systolic and diastolic parts. In this article, we propose to analyse the parameters computed from this technique in different clinical and physiological conditions. Two cases are considered : moderate chronic heart failure and high fit triathlets. The variability of these new parameters is compared to the variability of classical blood pressure parameters. Promising results are obtained.
\end{abstract}

\section{INTRODUCTION}

The analysis of mean values and beat-to-beat variability of cardiovascular (CV) time series has been widely used as a non invasive approach to study the control of the autonomic nervous system (ANS) on the CV function [1], [8]. Two main frequency bands are related to autonomic activity : an high frequency (HF), related to breathing activity, and a low frequency (LF), around $0.1 \mathrm{~Hz}$. The usual CV time series are the cardiac period ( RR interval) or the systolic period (SP), the systolic and diastolic pressures (SBP \& DBP). Standard measures are mean levels and global variability, spectral and time-frequency analysis such as the smoothed pseudo Wigner-Ville distribution (SPWVD), local harmonic analysis (the Complex Demodulation) [10] and the spectral RR/SBP gain. The general functioning of the ANS is relatively well described by these parameters in obvious physiological or pathological situations. But the early diagnosis of a degradation/improvement in pathological or physiological cases requires very discriminating indices.

In a recent article [7], an original Scattering-Based Signal Analysis (SBSA) method was proposed. The latter was applied in [6] for reconstructing the Arterial Blood Pressure (ABP) waves and separating its systolic and diastolic parts, an application that can be of great interest. This approach is based on the scattering transform and consists in solving the spectral problem of a one dimensional Schrödinger equation [2], [3], [4] with a potential depending linearly upon the ABP signal [7]. This potential is then expressed with the discrete spectrum including negative eigenvalues and corresponds in fact to the interacting components of an N-soliton [5], [11].

The aim of this study is to compare the SBSA parameters to classical $\mathrm{CV}$ parameters, and this, in two non

T.M. Laleg, C. Médigue and M. Sorine are with INRIA-Rocquencourt, B.P. 105, 78153 Le Chesnay cedex, France.

taous-meriem.laleg@inria.fr,

claire.mediguedinria.fr,

michel.sorinedinria.fr.

F. Cottin is with Department of Sport and Exercise Science, University of Evry, Boulevard F. Mitterrand, 91025 Evry cedex, France. francois.cottin@bp.univ-evry.fr. obvious situations : discrimination between healthy subjects and moderate Chronic Heart Failure $(\mathrm{CHF})$ patients for the pathological situation; discrimination between two states for the physiological situation : High Fit Triathlets (HFT) before and after training. On top of classical variabilities (global, HF, LF), a third very high frequency (VHF) activity, near beat-to-beat variability was studied as its amplitude appears non negligible in the SBSA parameters.

In the next section, we present the experimental protocol. Then in section III, we introduce the SBSA parameters that will be analyzed. The results of the analysis are presented in section IV, followed by a discussion in section V. Finally, a conclusion summarizes the study.

\section{EXPERIMENTAL PROTOCOL}

The two pathological and physiological studies presented similar contexts : subjects were quiet, in supine position, under paced-breathing conditions. As breathing has a great influence on cardiovascular variability, this protocol presents the advantage to limit breathing in a narrow frequency band, homogeneous for all subjects. Non invasive blood pressure, sampled at $500 \mathrm{~Hz}$, was continuously recorded at the finger with a photoplethysmographic transducer ( $\mathrm{Fi}$ napres/Finometer) [13]. One/two minutes periods were selected in each recording, for computing mean levels and global variability (estimated by standard-deviation) from the classical parameters (SP, SBP and DBP) and the SBSA parameters that will be introduced in the next section. Results were expressed as means and Standard Error to Mean (SEM). Statistical analysis was made with non parametric tests : Mann-Whitney test for unpaired comparisons, Wilcoxon test for paired comparisons. For significance $*: P<0.05$; ** : $P<0.01$.

a) CHF patients versus healthy subjects: The study included nine moderate CHF patients, NYHA class II/III, and nine healthy control subjects, from 35 to 50 years old. For each subject, mean values and global variability were computed over a period of one minute, under $0.15 \mathrm{~Hz}$ pacedbreathing. The two groups were compared using the MannWhitney test.

b) HFT before and after training: The study included eight junior HFT, from 15 to 19 years old, recorded before training and five months later, after training, during competition. For each subject, mean values and global variability were computed over a period of two minutes under $0.25 \mathrm{~Hz}$ paced-breathing, before and after training. On top of mean values and global variability, the amplitudes in LF, $[0.03-0.15] \mathrm{Hz}, \mathrm{HF}, 0.25 \mathrm{~Hz}$, centered around the breathing frequency $([0.15-0.3] \mathrm{Hz})$, and $\mathrm{VHF},[0.3-0.6] \mathrm{Hz}$ were also 
computed using the SPWVD. The two situations were then compared using the Wilcoxon test.

\section{NEW BLOOD PRESSURE PARAMETERS}

In this section, we introduce the SBSA parameters that we propose to analyse. For more details about the SBSA method, the reader can refer to [6], [7]. We recall that the main idea in the SBSA approach consists in solving the spectral problem for a one dimensional Schrödinger equation :

$$
-\frac{\partial^{2} \psi}{\partial t^{2}}+V \psi=\lambda \psi
$$

where $\lambda$ and $\psi$ are respectively the eigenvalues and the eigenfunctions of the Schrödinger operator. $V$ is the Schrödinger potential given by :

$$
V(\chi)=-\chi P(t)
$$

$\chi$ is a positive parameter to determine and $P(t)$ the ABP signal. The idea is then to find $\chi$ that enables us to approximate the potential with its discrete spectrum only including negative eigenvalues. The ABP signal is then approximated by :

$$
\hat{P}(t)=4 \chi^{-1} \sum_{n=1}^{N} \kappa_{n} \psi_{n}^{2}(t),
$$

where $\lambda_{n}=-\kappa_{n}^{2}, n=1, \cdots, N$ are the $N$ negative eigenvalues and $\psi_{n}, n=1 \cdots, N$ the associated $L^{2}$-normalized eigenfunctions. Each component $4 \kappa_{n} \psi_{n}^{2}$ of the previous sum refers to a single soliton [5], [7]. Only 5-10 components are sufficient for a good reconstruction of the ABP [7].

Therefore, the first parameters that seem to be interesting to analyse are the negative eigenvalues. In this study, we restrict the analysis to the first two negative eigenvalues. However, there are other parameters that can contain meaningful physiological information for example the conserved quantities (or invariants), related to an $\mathrm{N}$-soliton solution of a Korteweg-de Vries equation [5], [9]. We consider here the first two invariants which are related to the $\kappa_{n}, n=1, \cdots, N$ by the following expressions :

$$
I N V_{1}=4 \chi^{-1} \sum_{n=1}^{N} \kappa_{n}, \quad I N V_{2}=\frac{16}{3} \chi^{-2} \sum_{n=1}^{N} \kappa_{n}^{3} .
$$

We point out that $I N V_{1}$ and $I N V_{2}$ represent respectively the mean $\mathrm{ABP}$ and the pressure momentum.

As introduced in [6], [7], the SBSA technique can be used to separate the systolic and diastolic parts of the ABP. For each phase, we can define the corresponding invariants. Then, the two first systolic invariants are :

$$
I N V S_{1}=4 \chi^{-1} \sum_{n=1}^{2} \kappa_{n}, \quad I N V S_{2}=\frac{16}{3} \chi^{-2} \sum_{n=1}^{2} \kappa_{n}^{3},
$$

and the two first diastolic invariants are given by :

$$
I N V D_{1}=I N V_{1}-I N V S_{1}, \quad I N V D_{2}=I N V_{2}-I N V S_{2} .
$$

As an illustration, Fig. 1 and Fig. 2 show time series of the classical and SBSA parameters, extracted from our two studies. The breathing influence appears clearly in the two
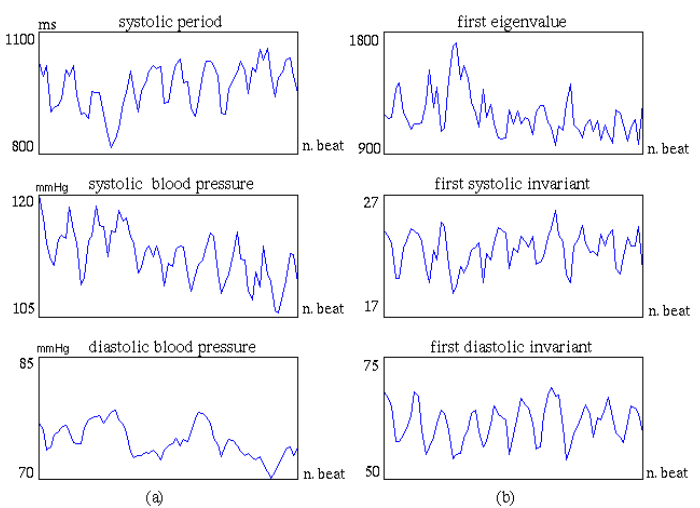

Fig. 1. Time series of ABP parameters in a healthy subject, under $0.15 \mathrm{~Hz}$ paced breathing, during one minute analysis. Direct classical parameters in (a) and three of the SBSA parameters in (b).
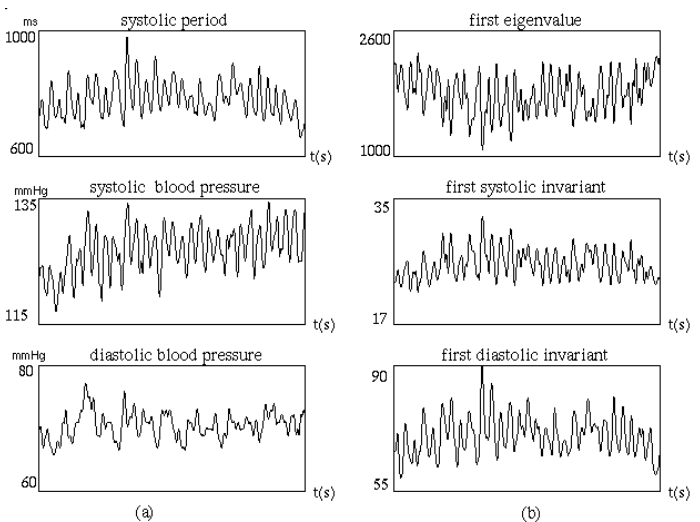

Fig. 2. Time series of ABP parameters in a healthy subject, under $0.25 \mathrm{~Hz}$ paced breathing, during two minutes analysis. Direct classical parameters in (a) and three of the SBSA parameters in (b).

cases. Fig. 3 points out the VHF, particularly important on the SBSA parameters, such as on the eigenvalues. The latter are effected by any change in the $\mathrm{ABP}$ waveform.

\section{RESULTS}

\section{A. CHF patients versus healthy subjects}

Mean levels of the parameters are not significantly different between control and CHF subjects. However, while the global variability is not significant for the classical parameters, it is significantly lower for the SBSA parameters (Table I). Two representative time series of $\left|\lambda_{1}\right|, I N V S_{1}$ and $I N V D_{1}$ are shown in Fig. 4.

\section{B. HFT subjects before and after training}

As shown in Table II, mean levels of the parameters are not significantly different between the two physiological situations : before and after training. The global variability of the parameters is also not significantly different except for the first normalized systolic and diastolic invariants (normalized by the total variability of the first invariant). Then, we did a deeper analysis of the systolic and diastolic invariants variability, by extracting HF, LF and VHF components. A 
TABLE I

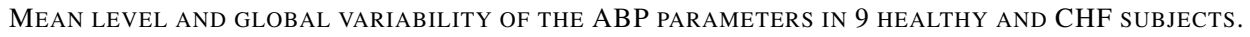

\begin{tabular}{lccccc}
\hline \multirow{2}{*}{ ABP parameters } & \multicolumn{2}{c}{ Mean level } & & \multicolumn{2}{c}{ Global variability } \\
\cline { 2 - 3 } \cline { 5 - 6 } Systolic period & Healthy subjects & CHF patients & & Healthy subjects & CHF patients \\
Systolic blood pressure & $804 \pm 31$ & $887 \pm 51$ & & $50.18 \pm 9.244$ & $25.00 \pm 3.494$ \\
Diastolic blood pressure & $113.3 \pm 4.9$ & $111.8 \pm 6.2$ & & $4.286 \pm 0.549$ & $3.254 \pm 0.301$ \\
First eigenvalue & $65.6 \pm 4.3$ & $63.9 \pm 4.1$ & & $2.110 \pm 0.422$ & $1.348 \pm 0.175$ \\
Second eigenvalue & $1898 \pm 149$ & $1683 \pm 265$ & & $256.4 \pm 44.30$ & $140.2 \pm 16.91 * *$ \\
First invariant & $1556 \pm 114$ & $1343 \pm 227$ & & $214.0 \pm 37.53$ & $114.0 \pm 15.66 * *$ \\
Second invariant & $65.5 \pm 4.3$ & $68.5 \pm 4.5$ & & $4.778 \pm 0.649$ & $2.492 \pm 0.262^{*}$ \\
First systolic invariant & $5648 \pm 618$ & $5670 \pm 648$ & & $534.9 \pm 95.7$ & $283.7 \pm 30.08 * *$ \\
Second systolic invariant & $17.7 \pm 1.1$ & $18.7 \pm 1.3$ & & $1.447 \pm 0.192$ & $0.916 \pm 0.064^{* *}$ \\
First diastolic invariant & $2196 \pm 229$ & $2253 \pm 273$ & & $230.3 \pm 41.26$ & $136.7 \pm 12.06 *$ \\
Second diastolic invariant & $47.4 \pm 3$ & $47.9 \pm 3.3$ & & $3.424 \pm 0.47$ & $1.940 \pm 0.203 * *$ \\
\hline & $3255 \pm 324$ & $3362 \pm 384$ & & $307.5 \pm 56.59$ & $175.0 \pm 25.31 * *$ \\
\hline
\end{tabular}

Data are expressed as means and SEM, * $: P<0.05 ; * *: P<0.01$.

TABLE II

MEAN LEVEL AND GLOBAL VARIABILITY OF THE ABP PARAMETERS IN 8 JUNIOR HFT BEFORE AND AFTER TRAINING.

\begin{tabular}{lccccc}
\hline & \multicolumn{2}{c}{ Mean level } & & \multicolumn{2}{c}{ Global variability } \\
\cline { 2 - 3 } \cline { 5 - 6 } ABP parameters & Before training & After training & & Before training & After training \\
\hline Systolic period & $124.2 \pm 32 \pm 10.2$ & $935 \pm 46$ & & $69.67 \pm 8.466$ & $65.04 \pm 4.962$ \\
Systolic blood pressure & $64.6 \pm 5.9$ & $51.3 \pm 5.3$ & & $5.460 \pm 0.756$ & $7.074 \pm 0.929$ \\
Diastolic blood pressure & $1524 \pm 97$ & $1487 \pm 158$ & & $3.385 \pm 0.526$ & $3.538 \pm 0.429$ \\
First eigenvalue & $9810 \pm 931$ & $1161 \pm 115$ & & $210.9 \pm 20.40$ & $246.3 \pm 28.99$ \\
Second eigenvalue & $91.0 \pm 4.8$ & $83.3 \pm 4.6$ & & $8.104 \pm 1.393$ & $178.9 \pm 18.53$ \\
First invariant & $9810 \pm 931$ & $7880 \pm 703$ & & $1083.0 \pm 249.0$ & $91277 \pm 0.654$ \\
Second invariant & $24.7 \pm 1.2$ & $22.9 \pm 1.2$ & & $2.358 \pm 0.340$ & $2.160 \pm 0.18$ \\
First systolic invariant & $3777 \pm 303$ & $3154 \pm 257$ & & $434.6 \pm 83.34$ & $381.4 \pm 38.68$ \\
Second systolic invariant & $66.3 \pm 3.6$ & $60.3 \pm 3.3$ & & $5.963 \pm 1.038$ & $5.315 \pm 0.467$ \\
First diastolic invariant & $6032 \pm 637$ & $4725 \pm 449$ & & $667.0 \pm 165.6$ & $544.5 \pm 56.67$ \\
Second diastolic invariant & $0.272 \pm 0.002$ & $0.276 \pm 0.001$ & & $8.80 E+04 \pm 8.41 E 02$ & $9.02 E+04 \pm 7.52 E 02 *$ \\
First systolic normalized invariant & $0.388 \pm 0.008$ & $0.402 \pm 0.005$ & & $9.51 E+04 \pm 3.08 E 03$ & $9.98 E+04 \pm 3.15 E 03$ \\
Second systolic normalized invariant & $0.728 \pm 0.002$ & $0.724 \pm 0.001$ & & $8.80 E+04 \pm 8.41 E 02$ & $9.02 E+04 \pm 7.52 E 02 *$ \\
First diastolic normalized invariant & $0.612 \pm 0.008$ & $0.598 \pm 0.005$ & & $9.51 E+04 \pm 3.08 E 03$ & $9.98 E+04 \pm 3.15 E 03$ \\
Second diastolic normalized invariant & & & & &
\end{tabular}

TABLE III

VARIABILITY OF ABP INVARIANTS IN 3 FREQUENCY BANDS, BEFORE AND AFTER TRAINING IN 8 JUNIOR HFT.

\begin{tabular}{lcc}
\hline Mean amplitude in & Before training & After training \\
\hline HF & & \\
$\quad$ First systolic invariant & $2.132 \pm 0.246$ & $1.924 \pm 0.2$ \\
$\quad$ Second systolic invariant & $383.2 \pm 54.94$ & $305.4 \pm 35.58$ \\
$\quad$ First diastolic invariant & $5.202 \pm 0.723$ & $4.646 \pm 0.441$ \\
$\quad$ Second diastolic invariant & $529.9 \pm 96.08$ & $395.5 \pm 39.72$ \\
LF & & \\
$\quad$ First systolic invariant & $1.838 \pm 0.32$ & $1.699 \pm 0.192$ \\
$\quad$ Second systolic invariant & $324.6 \pm 61.01$ & $317.9 \pm 38.93$ \\
$\quad$ First diastolic invariant & $4.679 \pm 0.843$ & $4.111 \pm 0.513$ \\
$\quad$ Second diastolic invariant & $502.0 \pm 105.8$ & $461.8 \pm 59.07$ \\
VHF & & \\
$\quad$ First systolic invariant & $1.018 \pm 0.048$ & $0.814 \pm 0.052^{*}$ \\
$\quad$ Second systolic invariant & $149.1 \pm 11.54$ & $105.2 \pm 9.747^{*}$ \\
$\quad$ First diastolic invariant & $2.183 \pm 0.234$ & $1.555 \pm 0.109^{*}$ \\
$\quad$ Second diastolic invariant & $166.7 \pm 27.98$ & $97.71 \pm 9.367^{*}$ \\
\hline Data are expressed as means and SEM, $* P<0.05$. &
\end{tabular}

significant diminution appears only for the VHF amplitude (Table III, Fig. 5).

\section{DISCUSSION}

\section{A. CHF patients versus healthy subjects}

The results obtained in this study agree with previous ones obtained with the same data base [8] : lower variability in
CHF patients. In the previous study, the significance depended on RR and SBP together. RR global variability, LF-RR, HF-SBP were significant with $P<0.05$; the spectral RR/SBP gain alone was significant with $P<0.01$. However, it needs a coherence function greater than 0.5 to verify linearity between RR and SBP, and 2 minutes analysis periods. These constraints limited the number of available data (seven/six subjects in the previous study versus nine/nine in the present study). In this study, near all the first and second SBSA parameters of the ABP were significant with $p<0.01$. So, the SBSA parameters present the advantage of getting very significant differences between healthy and moderate CHF subjects; and this, with such a simple measure as global variability (standard-deviation) and on a short analysis period (one minute). This last point is important in the case of CHF, where patients present a lot of extra-systoles.

\section{B. HFT patients before and after training}

As the only significant difference on the first computations (means and global variability) depended on the global variability of the first systolic and diastolic normalized invariants, we were interested in their HF, LF and VHF components. Interestingly, only the VHF amplitude was significantly lower after training. A diminution in the beat-to-beat variability measured by the square root of the mean of the summed 

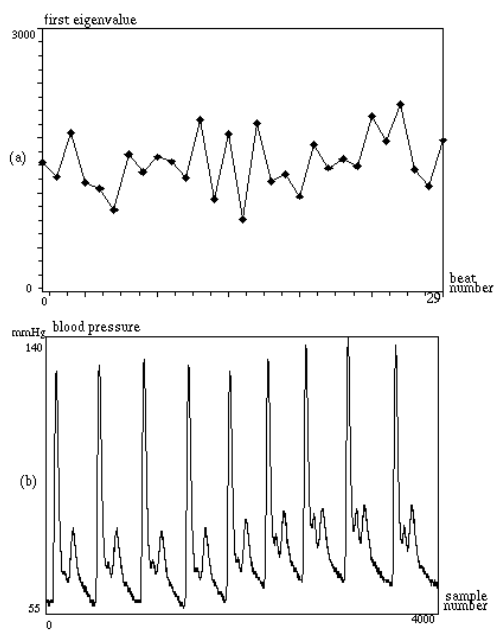

Fig. 3. (a) First eigenvalue time series of a healthy subject under $0.25 \mathrm{~Hz}$ paced-breathing during 29 beats. The signal exhibits a beat-to-beat variability, that is twice as fast as the breathing rate. (b) A zoom on a few beats shows morphological changes in the blood pressure signal.
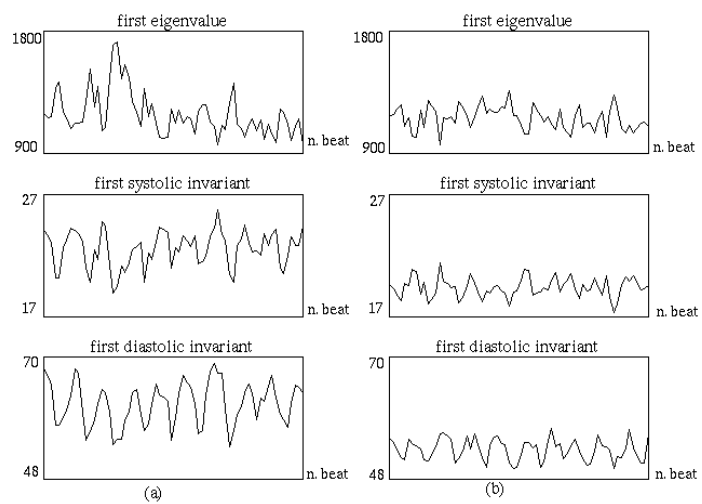

Fig. 4. Time series of the first blood pressure eigenvalues and invariants in, (a) a healthy and (b) a CHF subjects. The lower global variability in CHF is representative of the significant statistical difference over these two groups.

squared differences between adjacent beat-to-beat intervals in overtraining subjects was described in RR but not in the ABP [1]. A previous HF and LF analysis on RR-interval on the same data studied here (not published), as well as HF, LF, VHF on SP and SBP classical parameters did not show any significant differences. The aim of this kind of studies is to assess the quality of training, to avoid overtraining and to improve CV adjustment to exercise [1], [12].

\section{CONCLUSION}

In the two cases, CHF and HFT training, early indices to diagnosis and follow pathological and physiological situations is of great interest. New parameters have been introduced in this study to analyse the ABP signal. The results on the variability of these new $A B P$ indices are promising in particular which are related to their beat-tobeat variability. Moreover the SBSA parameters seem to contain meaningful physiological information, such as the
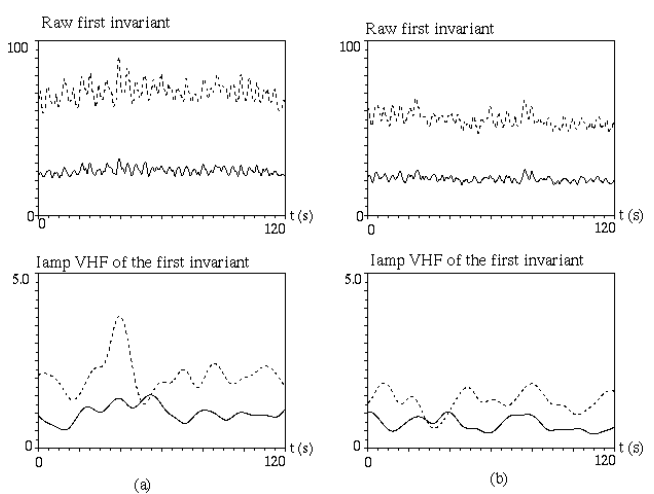

Fig. 5. First invariants of the ABP (top) and their VHF instantaneous amplitude (bottom) for a subject before (a) and after (b) training. Dotted lines stand for diastolic parameters, continuous lines stand for systolic ones. The decrease in VHF amplitude for diastolic and systolic invariants after training for this subject is representative of the significant statistical difference over the 8 subjects of the study.

first systolic invariant which is proportional to the stroke volume, difficult to measure routinely in a non invasive way.

\section{REFERENCES}

[1] M. Baumert, L. Brechtel, J. Lock, M. Hermsdorf, R. Wolff, V. Baier, and A. Voss, "Heart rate variability, blood pressure variability, and baroreflex sensitivity in overtrained athletes," Clin J Sport Med, vol. 15, no. 5, pp. 412-417, September 2006.

[2] F. Calogero and A. Degasperis, Spectral Transform and Solitons, J. Lions, G. Papanicolaou, R. Rockafellar, and H. Fujita, Eds. North Holland, 1982.

[3] P. A. Deift and E. Trubowitz, "Inverse scattering on the line," Commun. on Pure and Applied Mathematics, vol. XXXII, pp. 121-251, 1979.

[4] W. Eckhaus and A. Vanharten, The Inverse Scattering Transformation and the Theory of Solitons. North-Holland, 1983.

[5] C. S. Gardner, J. M. Greene, M. D. Kruskal, and R. M. Miura, "Korteweg-de vries equation and generalizations VI. Methods for exact solution," in Communications on pure and applied mathematics. J.Wiley \& sons, 1974, vol. XXVII, pp. 97-133.

[6] T. M. Laleg, E. Crépeau, Y. Papelier, and M. Sorine, "Arterial blood pressure analysis based on scattering transform I," in Proc. EMBC, Sciences and technologies for health, Lyon, France, August 2007.

[7] T. M. Laleg, E. Crépeau, and M. Sorine, "Travelling-wave analysis and identification. A scattering theory framework," in Proc. European Control Conference ECC, Kos, Greece, July 2007.

[8] L. Mangin, A. Monti, C. Médigue, I. Macquin-Mavier, M. E. Lopes, P. Gueret, A. Castaigne, B. Swynghedauw, and P. Mansier, "Altered baroreflex gain during voluntary breathing in chronic heart failure," European Journal of Heart Failure, vol. 3, pp. 189-195, 2001.

[9] R. M. Miura, "The korteweg-de vries equation : A survey of results," SIAM Rewiew, vol. 18, no. 3, pp. 412-459, July 1976.

[10] A. Monti, C. Médigue, and L. Mangin, "Instantaneous parameter estimation in cardiovascular time series by harmonic and time-frequency analysis," IEEE Transactions on Biomedical Engineering, vol. 49, no. 12, pp. 1547-1556, December 2002.

[11] M. Remoissenet, Waves called solitons, concepts and experiments, $3^{\text {rd }}$ ed. Springer, 1999.

[12] A. L. T. Uusitalo, A. J. Uusitalo, and H. K. Rusko, "Heart rate and blood pressure variability during heavy training and overtraining in the female athlete," Int J Sports Med, vol. 21, no. 1, pp. 45-53, 2000.

[13] K. H. Wesseling, "Method and device for controlling the cuff pressure in measuring the blood pressure in a finger by means of photo-electric plethysmograph," United State Patent, September 1985. 